\title{
Fatores associados ao excesso de peso em crianças brasileiras menores de cinco anos
}

\author{
Factors associated with excess weight \\ in Brazilian children under five years of age
}

Fernanda de Oliveira Meller ${ }^{1}$

Cora Luiza Pavin Araújo ${ }^{1}$

Samanta Winck Madruga ${ }^{2}$

${ }^{1}$ Programa de PósGraduação em Nutrição e Alimentos, Universidade Federal de Pelotas. Campus Universitário, Caixa Postal 354. 96.010-900 Pelotas RS Brasil.

fe_meller@hotmail.com ${ }^{2}$ Programa de Pós-

Graduação em Educação Física, Universidade Federal de Pelotas, Brasil.
Abstract The scope of the study was to identify factors associated with excess weight in Brazilian children less than five years of age. Data from a cross-sectional home-based study entitled the $\mathrm{Na}$ tional Demographic and Health Survey (NDHS) of 2006 were used. The diagnosis of excess weight was performed using the weight-for-height greater than $2 z$ scores above the median anthropometric standard recommended by the World Health Organization in 2006. The population under study consisted of 4,388 children. The prevalence of excess weight in children was described according to socio-economic, demographic, anthropometric, and behavioral variables. Crude and adjusted analyses were carried out to assess statistical significance stratified by gender using Poisson regression. The prevalence of excess weight was $6.6 \%$. After adjusting for potential confounding factors, maternal body mass index and birth weight were associated with excess weight. Furthermore, being overweight was associated with exclusive breastfeeding for boys and with the socio-economic level and marital status of the mother for girls. The need to implement public policies that act on the major determinants of excess weight since childhood is emphasized.

Key words Overweight, Determinants, Children, Cross-sectional study
Resumo O objetivo do estudo foi identificar os fatores associados ao excesso de peso em crianças brasileiras menores de cinco anos. Foram utilizados dados da Pesquisa Nacional de Demografia e Saúde (PNDS) de 2006, um estudo transversal de base domiciliar. O diagnóstico do excesso de peso baseou-se no indice peso-para-altura superior a 2 escores $z$ acima da mediana do padrão antropométrico da Organização Mundial de Saúde - 2006. A população estudada constituiu-se de 4.388 crianças. A prevalência de excesso de peso nas crianças foi descrita segundo variáveis socioeconômicas, demográficas, antropométricas e comportamentais. Foram realizadas análises bruta e ajustada através da Regressão de Poisson e todas foram estratificadas pelo sexo da criança. A prevalência de excesso de peso foi de 6,6\%. Após análise ajustada, o indice de massa corporal materno e o peso ao nascer foram associados ao excesso de peso. Além disso, o excesso de peso mostrou-se associado ao tempo de amamentação exclusiva, nos meninos e ao nivel socioeconômico e situação conjugal, nas meninas. Enfatiza-se a necessidade da implementação de políticas públicas que atuem, o mais precocemente possivel, nos principais determinantes do excesso de peso.

Palavras-chave Excesso de peso, Determinantes, Crianças, Estudo transversal 


\section{Introdução}

Importantes mudanças demográficas, econômicas, sociais e tecnológicas ocorridas nas últimas décadas propiciaram alterações importantes no padrão de morbimortalidade, inclusive nos indicadores nutricionais ${ }^{1}$.

Paralelamente à redução contínua dos casos de desnutrição, observa-se um incremento nas prevalências de excesso de peso que, por sua vez, aumenta o risco de doenças crônicas não transmissíveis ${ }^{2}$, sendo este processo conhecido como transição nutricional ${ }^{3}$.

Obesidade caracteriza-se pelo excesso de gordura corporal em relação à massa magra e tratase de uma doença multifatorial, determinada por fatores genéticos, comportamentais, ambientais e culturais ${ }^{4,5}$. Alguns autores têm mostrado que, na infância, o desenvolvimento do sobrepeso está associado, entre outros fatores, ao nível socioeconômico, escolaridade e sobrepeso materno, cor da pele, idade, sexo, peso ao nascer e tempo de amamentação ${ }^{6-8}$.

A obesidade infantil vem sendo considerada um importante problema de saúde pública9 ${ }^{9}$. No ano de 2010, estimou-se que 43 milhões de crianças menores de cinco anos de idade apresentavam sobrepeso e obesidade, sendo que a grande maioria vivia em países em desenvolvimento (35 milhões $)^{10}$. O impacto econômico global que essa doença poderá causar tem sido uma grande preocupação ${ }^{9}$, considerando-se que a persistência da obesidade na vida adulta pode resultar em formas mais graves da obesidade, acompanhadas de elevadas taxas de morbimortalidade ${ }^{11}$.

Em função da atual magnitude da obesidade e da velocidade da sua evolução em vários países do mundo, este agravo tem sido definido como uma pandemia, atingindo tanto países desenvolvidos como em desenvolvimento, entre eles o Brasil ${ }^{12,13}$.

A abordagem preventiva da obesidade é extremamente necessária e tem se mostrado efetiva quando implantada ao grupo etário infantil, visto que o processo patológico com início na infância pode se perpetuar na vida adulta, tornando mais difícil seu tratamento e controle ${ }^{14,15}$.

A infância é, portanto, o principal grupo-alvo para estratégias de prevenção e controle do sobrepeso, não só devido à sua característica como grupo de risco, mas também por conta da maior probabilidade de sucesso das ações a serem implementadas ${ }^{16}$. Segundo Guedes et al. ${ }^{17}$, a identificação de variáveis que melhor expliquem as prevalências de sobrepeso na população infantil poderá oferecer importantes subsídios para a implementação de medidas de intervenção.

Assim, o presente trabalho teve como objetivo identificar os fatores associados ao excesso de peso em crianças brasileiras menores de cinco anos estudadas na Pesquisa Nacional de Demografia e Saúde no ano de 2006, publicada em $2008^{18}$.

\section{Metodologia}

O estudo compreende um recorte da Pesquisa Nacional de Demografia e Saúde (PNDS) de $2006^{18}$. Este inquérito de âmbito nacional teve como objetivo central caracterizar a população feminina em idade fértil ( 15 a 49 anos) e as crianças menores de cinco anos, segundo fatores demográficos, socioeconômicos e culturais. Tratase de um estudo transversal, de base domiciliar, realizado entre os anos de 2006 e 2007.

O plano amostral da PNDS-2006 ${ }^{18}$ foi desenhado para fornecer estimativas representativas das mulheres brasileiras em idade fértil residentes em domicílios particulares em setores comuns ou não especiais (inclusive favelas), selecionados em dez estratos amostrais, incluindo áreas urbanas e rurais de todas as cinco grandes regiões geográficas brasileiras.

O estudo foi realizado por meio de uma amostra probabilística de domicílios obtida em dois estágios de seleção: as unidades primárias, que são os setores censitários, e as unidades secundárias, que são os domicílios particulares, ocupados ou não ocupados.

A amostra de setores foi obtida de forma independente por conglomerados, sendo que o total de setores em cada estrato foi obtido de forma a garantir um total de entrevistas que permitisse um número mínimo de coleta de sangue em crianças, segundo os percentuais de ocorrência de deficiência de vitamina A no sangue.

Os domicílios foram selecionados aleatoriamente dentro de cada setor, sendo doze domicílios por setor para responderem ao questionário completo, os quais foram denominados domicílios elegíveis por conterem pelo menos uma mulher de 15 a 49 anos de idade.

Ao final do trabalho de campo foram obtidas informações sobre 14.617 domicílios, dos quais 13.056 tinham pelo menos uma mulher elegível, totalizando 15.575 mulheres de 15-49 anos entrevistadas.

A todos os domicílios selecionados na amostra, foi aplicado o questionário Ficha do Domicí- 
lio, preferencialmente ao responsável e, na ausência deste, a um morador maior de quinze anos de idade. Após, foi aplicado o Questionário da $\mathrm{Mu}$ lher a todas aquelas de 15 a 49 anos de idade identificadas como moradoras habituais da residência.

Após a aplicação dos questionários, foram mensurados o peso e a altura de mulheres e crianças e a circunferência da cintura (CC) de mulheres de acordo com as recomendações da $\mathrm{OMS}^{19}$. Essas medidas foram feitas duas vezes para cada pessoa, sendo calculada a média aritmética de ambas. A medida do peso foi obtida empregando-se balança eletrônica portátil da marca Dayhome ${ }^{\circledR}$, com capacidade de $150 \mathrm{Kg}$ e precisão de $0,1 \mathrm{Kg}$.

Em menores de dois anos foi medido o comprimento em um infantômetro, com a criança na posição deitada. Nas crianças com idade igual ou superior a dois anos e nas mulheres, a medida foi tomada na posição em pé, em um estadiômetro. Os infantômetros e os estadiômetros portáteis e as fitas de inserção para mensuração da CC foram desenvolvidos especialmente para a PNDS$2006^{18}$ no Laboratório de Avaliação Nutricional de Populações (LANPOP) do Departamento de Nutrição da Universidade de São Paulo. Os infantômetros apresentavam extensão de $110 \mathrm{~cm}$ e precisão de $0,1 \mathrm{~cm}$ e os estadiômetros, $210 \mathrm{~cm}$ e $0,1 \mathrm{~cm}$, respectivamente.

A mensuração da CC foi realizada no ponto médio entre a última costela e a crista ilíaca e, para essa medida, foi utilizada fita de inserção inextensível com 3,3 cm de largura, capacidade de $150 \mathrm{~cm}$ e precisão de $0,1 \mathrm{~cm}$. Todos os aparelhos utilizados foram calibrados no início e no final de cada dia de trabalho.

As informações foram coletadas para todas as crianças menores de cinco anos que se encontravam na casa e que eram filhas das mulheres elegíveis. Portanto, as variáveis das mães se repetem no caso de terem mais de um filho.

O programa utilizado para a entrada de dados foi o CSPro (Census and Survey Processing System) software desenvolvido pelo Bureau do Censo Norte-Americano ${ }^{20}$. O relatório final e a metodologia detalhada da PNDS-2006 estão publicamente disponibilizados ${ }^{18}$.

Foram identificadas 4.957 crianças menores de cinco anos de idade, sendo que para 4.415 dispunha-se de medidas de peso e altura. No presente estudo, optou-se por descartar valores extremos do índice peso-para-altura (menores que -6 escores $z$ e maiores que +6 escores $z$ ) de acordo com a $\mathrm{OMS}^{19}$, assim, foram incluídas 4.388 crianças na presente análise.
O desfecho foi dicotomizado em "sem excesso de peso" e "com excesso de peso" e o critério para excesso de peso nas crianças menores de cinco anos baseou-se no índice peso-para-altura superior a 2 escores $\mathrm{z}$ acima da mediana do padrão antropométrico da $\mathrm{OMS}^{21}$.

As variáveis independentes gerais incluídas na análise foram macrorregião de moradia, situação de domicílio (urbano/rural) e nível socioeconômico. Para determinação do nível econômico, foi utilizada a classificação da Associação Brasileira de Empresas de Pesquisa (ABEP) do ano de $2011^{22}$ e no caso da falta de informação sobre os anos de estudo dos responsáveis pelos domicílios, foi utilizada a informação referente aos anos de estudo dos cônjuges ou companheiros. Esta variável foi recategorizada em A-B, C, D-E.

As variáveis independentes relacionadas à mãe foram escolaridade (em anos completos), trabalho fora do lar (sim/não), idade (em anos), situação conjugal (sem companheiro/com companheiro), cor da pele (informada pela respondente e dicotomizada em "branca" e "não branca”), índice de massa corporal (IMC) e CC. Em relação ao IMC, os valores inferiores a 16,0 Kg/ $\mathrm{m}^{2}$ foram excluídos ${ }^{19}$. Foram consideradas desnutridas, eutróficas, com sobrepeso e obesas, as mulheres com 20 anos de idade ou mais que apresentaram valor de IMC $<18,5 \mathrm{Kg} / \mathrm{m}^{2}$, entre 18,5 e $24,9 \mathrm{Kg} / \mathrm{m}^{2}$, entre 25,0 e $29,9 \mathrm{Kg} / \mathrm{m}^{2}$ e $\geq 30,0 \mathrm{Kg} /$ $\mathrm{m}^{2}$, respectivamente ${ }^{19}$. Para as adolescentes, foi utilizado o IMC-para-idade, sendo consideradas desnutridas, eutróficas, com sobrepeso e obesas aquelas que apresentaram IMC-para-idade inferior a -2 escores $z, \geq-2$ e $\leq+1$ escores $z,>$ $+1 \mathrm{e} \leq+2$ escores $\mathrm{z}$ e superior a +2 escores $\mathrm{z}$, respectivamente ${ }^{23}$. Quanto à CC, essa foi avaliada nas mulheres a partir de 20 anos de idade e categorizada em $<80 \mathrm{~cm}, 80$ a $87 \mathrm{~cm} \mathrm{e} \geq 88 \mathrm{~cm}^{19}$.

As variáveis independentes relacionadas à criança foram sexo, idade (em meses), tempo de amamentação exclusiva (em meses) e peso ao nascer (em gramas). A informação sobre o peso ao nascer foi obtida na caderneta da criança ou referida pela mãe. Esta segunda alternativa foi utilizada quando não se localizava a caderneta ou não havia registro da informação. A variável foi categorizada em $<3.000 \mathrm{~g}, 3.000$ a 3.999g e $\geq$ $4.000 \mathrm{~g}$ tendo em vista a baixa prevalência de excesso de peso nas crianças do sexo masculino nascidas com peso inferior a $2.500 \mathrm{~g}$. A permanência desta categoria de peso ao nascer (< $2.500 \mathrm{~g}$ ) inviabilizaria a análise com essa variável.

As variáveis independentes contínuas foram categorizadas a fim de observar tendência em 
relação ao desfecho. Para as demais variáveis, utilizaram-se as categorias originais da PNDS$2006^{18}$.

Os dados foram analisados no programa estatístico Stata (versão 11.0), utilizando o comando svyset para definir os pesos amostrais e conglomerados e o prefixo svy em todas as análises realizadas, tendo em vista a complexidade do processo de amostragem.

A análise bruta foi conduzida a fim de conhecer a prevalência de excesso de peso conforme os diferentes grupos de variáveis independentes. Para a avaliação da significância estatística, foi utilizada Regressão de Poisson, apresentando-se o valor p correspondente ao teste de Wald para heterogeneidade ou tendência linear para variáveis categóricas ordinais. A análise multivariável também foi realizada a partir de Regressão de Poisson, respeitando o modelo de análise (Figura 1) ${ }^{24}$. Todas as variáveis fizeram parte da análise e aquelas com valor $\mathrm{p}$ menor que 0,20 permaneceram no modelo de regressão como possíveis fatores de confusão.

Todas as análises foram estratificadas pelo sexo da criança.

O presente estudo foi aprovado pelo Comitê de Ética em Pesquisa do Centro de Referência e Treinamento DST/AIDS de São Paulo.

\section{Resultados}

A PNDS-2006 ${ }^{18}$ entrevistou 15.575 mulheres na faixa etária entre 15 e 49 anos de idade, residentes em 13.056 domicílios visitados. Nestes, foram identificadas 4.957 crianças menores de cinco anos, sendo que para 4.415 foi calculado o índice peso-para-altura. No presente estudo, foram incluídas 4.388 crianças, após exclusão de valores extremos de escore $\mathrm{z}$ do índice peso-para-altura, representando 12.392 .428 crianças brasileiras menores de cinco anos.

As características das crianças e das mães são apresentadas na Tabela 1 . Observa-se que a maioria das crianças era do sexo masculino $(52,2 \%)$, cerca de $1 / 3$ foi amamentada por, no máximo, dois meses, $26 \%$ das crianças nasceram com peso inferior a $3.000 \mathrm{~g}$, sendo que $6 \%$ destas apresentaram peso ao nascer inferior a $2.500 \mathrm{~g}$ (4,5\% dos meninos e 7,6\% das meninas), dados não apresentados na tabela. Quanto ao estado nutricional, 6,6\% das crianças apresentaram excesso de peso. Com relação às mães, observa-se que $89 \%$ pertenciam a famílias de classes C, D ou E e mais da metade apresentaram faixa etária entre 20 e
29 anos (56\%). As prevalências de desnutrição e excesso de peso (sobrepeso e obesidade) foram cerca de $4 \%$ e de $43 \%$, respectivamente, enquanto que $29 \%$ apresentaram CC igual ou superior a $88 \mathrm{~cm}$.

Na Tabela 2, estão apresentadas as prevalências de excesso de peso entre as crianças, segundo as variáveis independentes incluídas no estudo e estratificadas por sexo. As variáveis peso ao nascer, trabalho fora do lar e cor da pele da mãe mostraram associação significativa com o excesso de peso nos meninos, nas meninas e na amostra total. As associações com o peso ao nascer nos meninos e na amostra total apresentaram tendência linear positiva, ou seja, quanto maior o peso ao nascer maiores as prevalências de excesso de peso $(p<0,001)$. Crianças filhas de mães que trabalhavam fora do lar apresentaram maiores prevalências de excesso de peso. Quanto à associação com a cor da pele, as crianças cujas mães referiram ter cor da pele branca foram as que apresentaram maiores prevalências de excesso de peso.

Com relação ao tempo de amamentação exclusiva, houve associação apenas entre os meninos, enquanto que o nível socioeconômico mostrou-se inversamente associado ao excesso de peso somente nas meninas e na amostra total. Evidenciou-se associação com o IMC das mães na amostra total e entre os meninos. As demais variáveis não foram significativamente associadas ao excesso de peso nas crianças (Tabela 2).

A Tabela 3 mostra as associações brutas e ajustadas entre o excesso de peso e as variáveis independentes da amostra conforme o sexo. Após análise ajustada para possíveis fatores de confusão, o peso ao nascer permaneceu associado ao excesso de peso, sendo observada uma tendência linear positiva, tanto nos meninos ( $p<0,001)$ quanto nas meninas $(\mathrm{p}<0,001)$. A probabilidade de apresentar excesso de peso foi cerca de 7,5 e 4,0 vezes maior nos meninos e meninas, respectivamente, que nasceram com peso igual ou superior a $4.000 \mathrm{~g}$ em relação aos que nasceram com menos de 3.000g. Quanto à variável tempo de amamentação exclusiva, esta permaneceu associada ao excesso de peso nos meninos, sendo que aqueles que foram amamentados por um período de 5 a 6 meses apresentaram menor probabilidade de terem excesso de peso. O IMC da mãe mostrou associação com o excesso de peso em ambos os sexos, sendo que as prevalências de excesso de peso foram mais elevadas naquelas crianças cujas mães eram obesas. A associação entre o nível socioeconômico e o excesso de peso 
Variáveis da mãe

Variáveis da criança

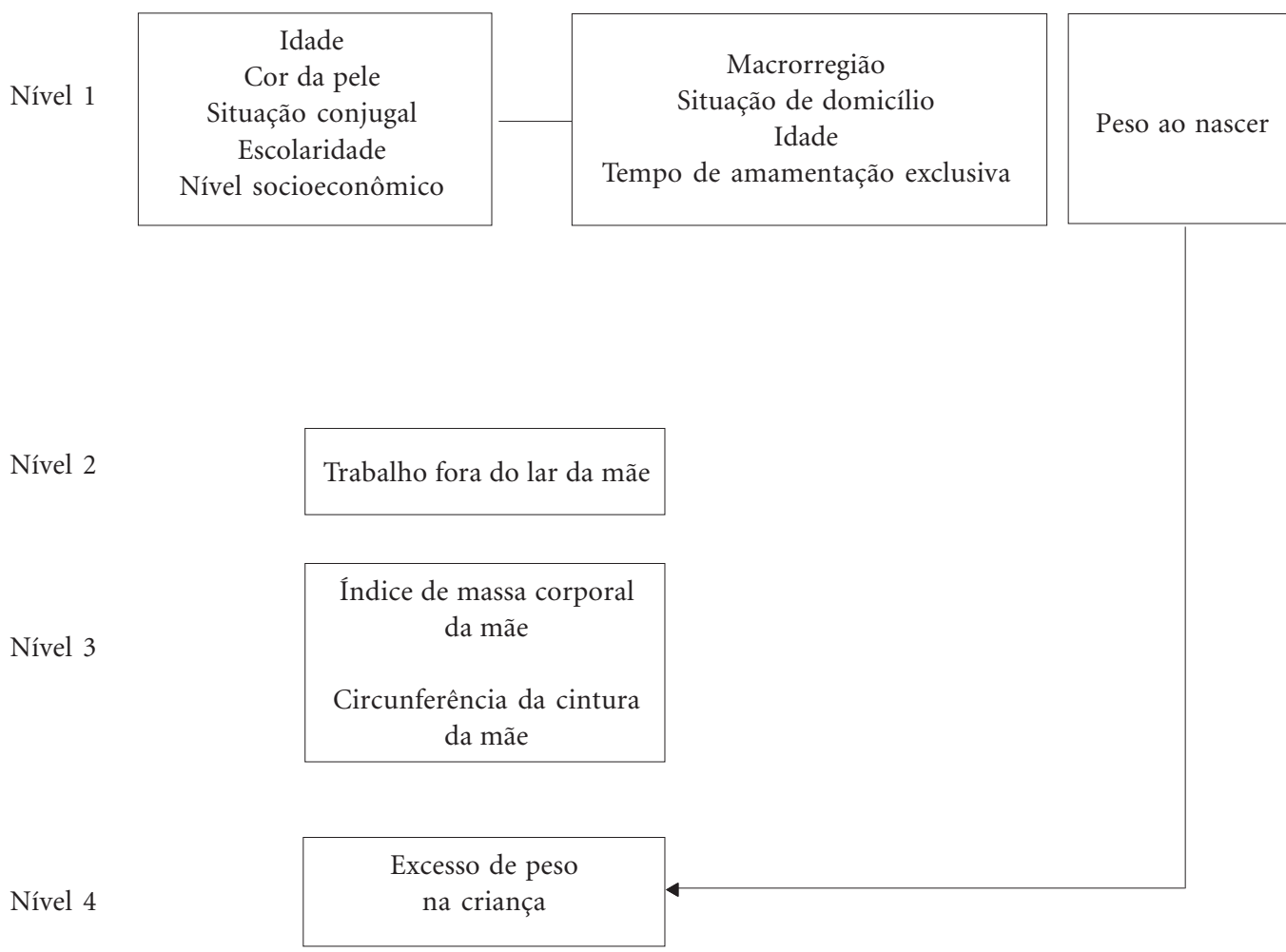

Figura 1. Modelo de análise dos fatores associados ao excesso de peso nas crianças.

permaneceu significativa apenas nas meninas, sendo observada uma tendência linear inversa. Observou-se que a situação conjugal esteve associada ao excesso de peso nas meninas, sendo que aquelas cujas mães viviam com companheiros apresentaram maior probabilidade de terem excesso de peso. A cor da pele da mãe e o trabalho fora do lar perderam a associação com o excesso de peso em ambos os sexos após o ajuste. As demais variáveis permaneceram não associadas ao excesso de peso.

\section{Discussão}

Um importante achado neste estudo foi a associação entre o peso ao nascer e o excesso de peso nos meninos e nas meninas, tanto na análise bruta quanto na ajustada. Este resultado foi consis- tente com estudos realizados em países desenvolvi$\operatorname{dos}^{7,25}$. Oldroyd et al. ${ }^{26}$ evidenciaram que o excesso de peso nas crianças com faixa etária entre quatro e cinco anos esteve associado àquelas que nasceram com peso superior a $4.000 \mathrm{~g}$. Tal associação sugere que a situação nutricional intrauterina, refletida pelo peso ao nascer, pode ser determinante importante não só da sobrevivência infantil como, também, do estado nutricional nos primeiros anos de vida ${ }^{27}$. Segundo Chaparro et al. ${ }^{28}$, quanto maior o peso ao nascer, menor a probabilidade da mãe classificar corretamente o estado nutricional da criança e, consequentemente, tal fato predispõe ao excesso de peso na infância.

Observou-se maior prevalência de excesso de peso nas crianças cujas mães eram obesas, após a análise ajustada. Resultados semelhantes foram encontrados por $\mathrm{Gewa}^{8}$ ao analisar dados da Pesquisa de Demografia e Saúde no Quênia, o 
Tabela 1. Distribuição da amostra estudada de acordo com características socioeconômicas, demográficas, comportamental e antropométricas $(n=4.388$ crianças; $n=3.682$ mães). Pesquisa Nacional de Demografia e Saúde, 2006.

\begin{tabular}{|c|c|c|c|c|c|}
\hline Variáveis & $\mathbf{n}$ & $\%$ & Variáveis & $\mathbf{n}$ & $\%$ \\
\hline Macrorregião & & & Nível socioeconômico & & \\
\hline Norte & 980 & 10,6 & $\mathrm{~A}-\mathrm{B}$ & 303 & 11,3 \\
\hline Nordeste & 865 & 28,2 & $\mathrm{C}$ & 1110 & 43,4 \\
\hline Sudeste & 869 & 41,4 & $D-E$ & 1363 & 45,3 \\
\hline Sul & 803 & 12,2 & \multicolumn{3}{|c|}{ Escolaridade da mãe (anos completos) } \\
\hline Centro-oeste & 871 & 7,6 & 0 a 4 & 862 & 18,0 \\
\hline Situação de domicílio & & & 5 a 8 & 1321 & 36,4 \\
\hline Urbana & 2868 & 80,8 & 9 a 11 & 1215 & 38,1 \\
\hline Rural & 1520 & 19,2 & 12 ou mais & 239 & 7,5 \\
\hline Sexo da criança & & & Trabalho fora do lar & & \\
\hline Masculino & 2251 & 52,2 & Sim & 1338 & 36,8 \\
\hline Feminino & 2137 & 47,8 & Não & 2342 & 63,2 \\
\hline Idade da criança (meses) & & & Idade da mãe (anos) & & \\
\hline 0 a 11 & 885 & 21,5 & $<20$ & 328 & 11,2 \\
\hline 12 a 23 & 857 & 19,3 & 20 a 29 & 2053 & 56,0 \\
\hline 24 a 35 & 874 & 19,6 & 30 a 34 & 731 & 17,7 \\
\hline 36 a 47 & 897 & 20,2 & 35 ou mais & 570 & 15,1 \\
\hline 48 a 59 & 875 & 19,4 & Situação conjugal & & \\
\hline Tempo de amamentação exclusiva & & & Sem companheiro & 561 & 14,8 \\
\hline (meses) & & & Com companheiro & 3119 & 85,2 \\
\hline$<1$ & 859 & 20,5 & Cor da pele da mãe & & \\
\hline 1 a 2 & 711 & 15,9 & Branca & 1287 & 34,4 \\
\hline 3 a 4 & 902 & 24,5 & Não branca & 2353 & 65,6 \\
\hline 5 a 6 & 1215 & 30,9 & Índice de massa corporal & & \\
\hline$>6$ & 274 & 8,2 & Desnutrição & 129 & 3,7 \\
\hline Peso ao nascer (gramas) & & & Eutrofia & 1969 & 52,9 \\
\hline$<3.000$ & 1159 & 25,6 & Sobrepeso & 1020 & 29,1 \\
\hline 3.000 a 3.999 & 2779 & 67,5 & Obesidade & 541 & 14,3 \\
\hline$\geq 4.000$ & 301 & 6,9 & Circunferência da cintura & & \\
\hline Excesso de peso da criança & & & $<80$ & 1529 & 46,6 \\
\hline $\operatorname{Sim}$ & 336 & 6,6 & 80 a 87 & 814 & 24,3 \\
\hline Não & 4052 & 93,4 & 88 ou mais & 971 & 29,1 \\
\hline
\end{tabular}

Percentual máximo de observações desconhecidas: 36,7\% (n=1612) para a variável nível socioeconômico.

qual evidenciou associação entre excesso de peso das mães e dos filhos, independente do sexo. Tal associação também foi evidenciada por outros autores $^{29,30}$. Em contrapartida, um estudo mostrou relação entre IMC materno e sobrepeso em meninas $^{31}$. A associação da obesidade infantil com o IMC da mãe pode ser devida à herança genética e/ou a fatores relacionados às condições ambientais. Estes fatores atuam, possivelmente, em conjunto, facilitando a expressão genética. $\mathrm{O}$ fato de a criança nascer em uma "família obesa" é fator de risco para que ela se torne obesa, isto porque, além da genética, os hábitos e comportamentos familiares proporcionam, especialmente para a criança, um ambiente favorável ao desenvolvimento da obesidade ${ }^{32}$.
Outro resultado que mostra a importância da mãe sobre o estado nutricional da criança é o efeito da situação conjugal. Para as meninas cujas mães viviam com companheiro, a prevalência de excesso de peso foi maior quando comparadas àquelas cujas mães viviam sem companheiro. Contudo, Zöllner e Fisberg ${ }^{27}$ não encontraram associação entre o excesso de peso em crianças e a situação conjugal da mãe. Já Kitsantas e Gaffney $^{7}$ observaram maior prevalência de excesso de peso nas crianças cujas mães não eram casadas.

Similarmente aos resultados encontrados no presente estudo, Morrissey et al. ${ }^{33}$, estudando crianças com faixa etária entre 8 e 10 anos, não observaram associação entre trabalho fora do 
Tabela 2. Prevalência de excesso de peso nas crianças segundo variáveis independentes na amostra total e estratificada por sexo $(n=4.388)$. Pesquisa Nacional de Demografia e Saúde, 2006.

\begin{tabular}{|c|c|c|c|c|c|c|c|c|c|}
\hline \multirow[b]{2}{*}{ Variáveis } & \multicolumn{3}{|c|}{ Masculino } & \multicolumn{3}{|c|}{ Feminino } & \multicolumn{3}{|c|}{ Total } \\
\hline & $\mathbf{n}$ & $\%$ & Valor $\mathrm{p}$ & $\mathbf{n}$ & $\%$ & Valor p & $\mathbf{n}$ & $\%$ & Valor $\mathbf{p}$ \\
\hline Macrorregião & & & $0,668^{\mathrm{b}}$ & & & $0,062^{\mathrm{b}}$ & & & $0,136^{\mathrm{b}}$ \\
\hline Norte & 489 & 6,4 & & 491 & 3,7 & & 980 & 5,1 & \\
\hline Nordeste & 452 & 5,9 & & 413 & 6,6 & & 865 & 6,2 & \\
\hline Sudeste & 430 & 6,5 & & 439 & 6,1 & & 869 & 6,3 & \\
\hline Sul & 420 & 8,5 & & 383 & 10,2 & & 803 & 9,3 & \\
\hline Centro-oeste & 460 & 5,6 & & 411 & 8,7 & & 871 & 7,0 & \\
\hline Situação de domicílio & & & $0,671^{\mathrm{b}}$ & & & $0,388^{\mathrm{b}}$ & & & $0,783^{b}$ \\
\hline Urbana & 1485 & 6,4 & & 1383 & 7,0 & & 2868 & 6,6 & \\
\hline Rural & 766 & 7,1 & & 754 & 5,6 & & 1520 & 6,3 & \\
\hline Idade da criança (meses) & & & $0,281^{\mathrm{b}}$ & & & $0,632^{\mathrm{b}}$ & & & $0,171^{\mathrm{b}}$ \\
\hline 0 a 11 & 449 & 7,4 & & 436 & 6,4 & & 885 & 6,9 & \\
\hline 12 a 23 & 465 & 6,5 & & 392 & 5,3 & & 857 & 6,0 & \\
\hline 24 a 35 & 456 & 5,8 & & 418 & 7,2 & & 874 & 6,4 & \\
\hline 36 a 47 & 462 & 8,8 & & 435 & 8,9 & & 897 & 8,9 & \\
\hline 48 a 59 & 419 & 3,7 & & 456 & 5,5 & & 875 & 4,6 & \\
\hline $\begin{array}{l}\text { Tempo de amamentação exclusiva } \\
\text { (meses) }\end{array}$ & & & $0,030^{\mathrm{b}}$ & & & $0,993^{\mathrm{b}}$ & & & $0,362^{\mathrm{b}}$ \\
\hline$<1$ & 434 & 3,5 & & 425 & 6,9 & & 859 & 5,1 & \\
\hline 1 a 2 & 361 & 7,4 & & 350 & 6,6 & & 711 & 7,0 & \\
\hline 3 a 4 & 450 & 10,2 & & 452 & 6,1 & & 902 & 8,1 & \\
\hline 5 a 6 & 644 & 4,1 & & 571 & 7,1 & & 1215 & 5,4 & \\
\hline$>6$ & 146 & 5,1 & & 128 & 5,8 & & 274 & 5,5 & \\
\hline Peso ao nascer (gramas) & & & $<0,001^{\mathrm{a}}$ & & & $0,043^{\mathrm{b}}$ & & & $<0,001^{\mathrm{a}}$ \\
\hline$<3.000$ & 518 & 2,2 & & 641 & 3,4 & & 1159 & 2,8 & \\
\hline 3.000 a 3.999 & 1462 & 7,6 & & 1317 & 8,1 & & 2779 & 7,8 & \\
\hline$\geq 4.000$ & 191 & 9,6 & & 110 & 7,2 & & 301 & 8,6 & \\
\hline Nível socioeconômico & & & $0,218^{\mathrm{b}}$ & & & $0,023^{\mathrm{a}}$ & & & $0,014^{\mathrm{a}}$ \\
\hline$A-B$ & 175 & 2,4 & & 190 & 1,7 & & 365 & 2,1 & \\
\hline $\mathrm{C}$ & 696 & 6,9 & & 616 & 6,2 & & 1312 & 6,6 & \\
\hline $\mathrm{D}-\mathrm{E}$ & 837 & 6,6 & & 806 & 7,6 & & 1643 & 7,1 & \\
\hline Escolaridade da mãe (anos completos) & & & $0,999^{\mathrm{b}}$ & & & $0,270^{\mathrm{b}}$ & & & $0,685^{\mathrm{b}}$ \\
\hline 0 a 4 & 565 & 6,7 & & 569 & 4,8 & & 1134 & 5,8 & \\
\hline 5 a 8 & 826 & 6,6 & & 749 & 7,4 & & 1575 & 6,9 & \\
\hline 9 a 11 & 706 & 6,6 & & 661 & 6,3 & & 1367 & 6,5 & \\
\hline 12 ou mais & 124 & 6,3 & & 133 & 10,6 & & 257 & 8,6 & \\
\hline Trabalho fora do lar & & & $0,024^{\mathrm{b}}$ & & & $0,055^{\mathrm{b}}$ & & & $0,003^{b}$ \\
\hline Sim & 767 & 8,9 & & 765 & 8,7 & & 1532 & 8,8 & \\
\hline Não & 1482 & 5,2 & & 1372 & 5,5 & & 2854 & 5,4 & \\
\hline Idade da mãe (anos) & & & $0,869^{\mathrm{b}}$ & & & $0,310^{\mathrm{b}}$ & & & $0,666^{\mathrm{b}}$ \\
\hline$<20$ & 193 & 6,1 & & 179 & 4,9 & & 372 & 5,5 & \\
\hline 20 a 29 & 1305 & 6,4 & & 1226 & 6,1 & & 2531 & 6,3 & \\
\hline 30 a 34 & 419 & 6,0 & & 414 & 10,1 & & 833 & 7,8 & \\
\hline 35 ou mais & 334 & 7,8 & & 318 & 6,6 & & 652 & 7,2 & \\
\hline Situação conjugal & & & $0,879^{\mathrm{b}}$ & & & $0,622^{\mathrm{b}}$ & & & $0,703^{b}$ \\
\hline Sem companheiro & 324 & 6,1 & & 317 & 5,8 & & 641 & 6,0 & \\
\hline Com companheiro & 1926 & 6,5 & & 1819 & 6,8 & & 3745 & 6,7 & \\
\hline Cor da pele da mãe & & & $0,039^{b}$ & & & $0,023^{\mathrm{b}}$ & & & $0,002^{\mathrm{b}}$ \\
\hline Branca & 764 & 8,8 & & 694 & 9,4 & & 1458 & 9,1 & \\
\hline Não branca & 1462 & 5,3 & & 1417 & 5,5 & & 2879 & 5,4 & \\
\hline Índice de massa corporal da mãe & & & $0,002^{\mathrm{b}}$ & & & $0,329^{\mathrm{b}}$ & & & $0,045^{b}$ \\
\hline Desnutrição & 75 & 0,1 & & 77 & 1,9 & & 152 & 1,1 & \\
\hline Eutrofia & 1215 & 6,5 & & 1144 & 6,6 & & 2359 & 6,6 & \\
\hline Sobrepeso & 630 & 6,5 & & 573 & 6,9 & & 1203 & 6,7 & \\
\hline Obesidade & 315 & 8,2 & & 330 & 7,3 & & 645 & 7,7 & \\
\hline Circunferência da cintura da mãe $(\mathrm{cm}$ & & & $0,484^{\mathrm{b}}$ & & & $0,840^{\mathrm{b}}$ & & & $0,847^{\mathrm{b}}$ \\
\hline$<80$ & 937 & 6,4 & & 882 & 6,6 & & 1819 & 6,5 & \\
\hline 80 a 87 & 494 & 5,4 & & 457 & 7,7 & & 951 & 6,5 & \\
\hline 88 ou mais & 592 & 7,7 & & 577 & 6,7 & & 1169 & 7,2 & \\
\hline
\end{tabular}

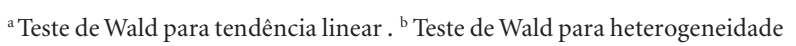


Tabela 3. Razão de prevalência bruta e ajustada da associação entre excesso de peso e variáveis independentes da amostra estratificada por sexo $(n=4.388)$. Pesquisa Nacional de Demografia e Saúde, 2006.

\begin{tabular}{|c|c|c|c|c|c|c|c|c|c|}
\hline \multirow[b]{2}{*}{ Nível } & \multirow[b]{2}{*}{ Variáveis } & \multicolumn{4}{|c|}{ Masculino } & \multicolumn{4}{|c|}{ Feminino } \\
\hline & & $\begin{array}{c}\text { RP } \\
\text { bruta(IC }\left(\mathrm{IC}_{95 \%}\right)\end{array}$ & $\begin{array}{l}\text { Valor } \\
\text { p }\end{array}$ & $\begin{array}{l}\text { RP ajustada }{ }^{b} \\
\left(\text { IC }_{95 \%}\right)\end{array}$ & $\begin{array}{c}\text { Valor } \\
\text { p }\end{array}$ & $\begin{array}{c}\text { RP } \\
\text { bruta(IC }\left(\mathrm{IC}_{95 \%}\right)\end{array}$ & $\begin{array}{c}\text { Valor } \\
\text { p }\end{array}$ & $\begin{array}{l}\text { RP ajustada }{ }^{b} \\
\left(\text { IC }_{95 \%}\right)\end{array}$ & $\begin{array}{c}\text { Valor } \\
\mathbf{p}\end{array}$ \\
\hline \multirow[t]{10}{*}{1} & Macrorregião & & $0,668^{\mathrm{b}}$ & & $0,749^{b}$ & & $0,062^{b}$ & & $0,366^{\mathrm{b}}$ \\
\hline & Norte & 1,00 & & 1,00 & & 1,00 & & 1,00 & \\
\hline & Nordeste & 0,92 & & 1,08 & & 1,79 & & 2,34 & \\
\hline & & $(0,51 ; 1,68)$ & & $(0,55 ; 2,13)$ & & $(0,88 ; 3,66)$ & & $(0,90 ; 6,09)$ & \\
\hline & Sudeste & 1,02 & & 1,24 & & 1,66 & & 1,41 & \\
\hline & & $(0,55 ; 1,89)$ & & $(0,61 ; 2,50)$ & & $(0,78 ; 3,55)$ & & $(0,50 ; 3,99)$ & \\
\hline & Sul & 1,34 & & 1,18 & & 2,75 & & 1,86 & \\
\hline & & $(0,73 ; 2,45)$ & & $(0,56 ; 2,48)$ & & $(1,34 ; 5,65)$ & & $(0,65 ; 5,32)$ & \\
\hline & Centro-oeste & 0,87 & & 0,80 & & 2,34 & & 2,24 & \\
\hline & & $(0,47 ; 1,63)$ & & $(0,39 ; 1,63)$ & & $(1,16 ; 4,73)$ & & $(0,85 ; 5,88)$ & \\
\hline \multirow[t]{4}{*}{1} & $\begin{array}{l}\text { Situação de } \\
\text { domicílio }\end{array}$ & & $0,671^{b}$ & & $0,687^{b}$ & & $0,388^{\mathrm{b}}$ & & $0,236^{\mathrm{b}}$ \\
\hline & Urbana & 1,00 & & 1,00 & & 1,00 & & 1,00 & \\
\hline & Rural & 1,11 & & 1,11 & & 0,80 & & 0,68 & \\
\hline & & $(0,69 ; 1,77)$ & & $(0,67 ; 1,86)$ & & $(0,48 ; 1,33)$ & & $(0,36 ; 1,28)$ & \\
\hline \multirow[t]{10}{*}{1} & $\begin{array}{l}\text { Idade da criança } \\
\text { (meses) }\end{array}$ & & $0,281^{b}$ & & $0,106^{\mathrm{b}}$ & & $0,632^{\mathrm{b}}$ & & $0,972^{\mathrm{b}}$ \\
\hline & 0 a 11 & 1,00 & & 1,00 & & 1,00 & & 1,00 & \\
\hline & 12 a 23 & 0,88 & & 1,10 & & 0,83 & & 0,80 & \\
\hline & & $(0,45 ; 1,72)$ & & $(0,45 ; 2,67)$ & & $(0,40 ; 1,76)$ & & $(0,30 ; 2,15)$ & \\
\hline & 24 a 35 & 0,78 & & 1,20 & & 1,13 & & 0,89 & \\
\hline & & $(0,38 ; 1,61)$ & & $(0,48 ; 3,03)$ & & $(0,58 ; 2,21)$ & & $(0,38 ; 2,07)$ & \\
\hline & 36 a 47 & 1,20 & & 1,59 & & 1,39 & & 0,81 & \\
\hline & & $(0,60 ; 2,41)$ & & $(0,64 ; 3,96)$ & & $(0,71 ; 2,71)$ & & $(0,32 ; 2,07)$ & \\
\hline & 48 a 59 & 0,51 & & 0,51 & & 0,85 & & 0,72 & \\
\hline & & $(0,23 ; 1,13)$ & & $(0,18 ; 1,47)$ & & $(0,42 ; 1,74)$ & & $(0,29 ; 1,81)$ & \\
\hline \multirow[t]{10}{*}{1} & $\begin{array}{l}\text { Tempo de } \\
\text { amamentação } \\
\text { exclusiva (meses) }\end{array}$ & & $0,030^{\mathrm{b}}$ & & $0,013^{b}$ & & $0,993^{b}$ & & $0,580^{\mathrm{b}}$ \\
\hline & $<1$ & 1,00 & & 1,00 & & 1,00 & & 1,00 & \\
\hline & 1 a 2 & 2,11 & & 2,81 & & 0,96 & & 1,03 & \\
\hline & & $(0,88 ; 5,03)$ & & $(1,13 ; 7,02)$ & & $(0,45 ; 2,04)$ & & $(0,40 ; 2,64)$ & \\
\hline & 3 a 4 & 2,91 & & 3,28 & & 0,88 & & 0,76 & \\
\hline & & $(1,34 ; 6,35)$ & & $(1,45 ; 7,43)$ & & $(0,41 ; 1,93)$ & & $(0,27 ; 2,15)$ & \\
\hline & 5 a 6 & 1,16 & & 1,32 & & 1,03 & & 1,56 & \\
\hline & & $(0,55 ; 2,48)$ & & $(0,59 ; 2,97)$ & & $(0,52 ; 2,04)$ & & $(0,72 ; 3,42)$ & \\
\hline & $>6$ & 1,46 & & 2,04 & & 0,85 & & 1,10 & \\
\hline & & $(0,52 ; 4,12)$ & & $(0,69 ; 6,03)$ & & $(0,31 ; 2,31)$ & & $(0,35 ; 3,48)$ & \\
\hline \multirow[t]{6}{*}{1} & $\begin{array}{l}\text { Peso ao nascer } \\
\text { (gramas) }\end{array}$ & & $<0,001^{\mathrm{a}}$ & & $<0,001^{\mathrm{a}}$ & & $0,043^{\mathrm{b}}$ & & $<0,001^{\mathrm{a}}$ \\
\hline & $<3.000$ & 1,00 & & 1,00 & & 1,00 & & 1,00 & \\
\hline & 3.000 a 3.999 & 3,19 & & 4,42 & & 2,40 & & 3,64 & \\
\hline & & $(1,52 ; 6,73)$ & & $(2,13 ; 9,17)$ & & $(1,21 ; 4,75)$ & & $(1,70 ; 7,81)$ & \\
\hline & $\geq 4.000$ & 3,83 & & 7,38 & & 2,14 & & 3,69 & \\
\hline & & $(1,52 ; 9,65)$ & & $(3,07 ; 17,72)$ & & $(0,73 ; 6,22)$ & & $(1,13 ; 12,07)$ & \\
\hline \multirow[t]{6}{*}{1} & $\begin{array}{l}\text { Nível } \\
\text { socioeconômico }\end{array}$ & & $0,218^{b}$ & & $0,767^{\mathrm{b}}$ & & $0,023^{\mathrm{a}}$ & & $0,016^{\mathrm{a}}$ \\
\hline & $\mathrm{A}-\mathrm{B}$ & 0,36 & & 0,69 & & 0,23 & & 0,24 & \\
\hline & & $(0,11 ; 1,19)$ & & $(0,20 ; 2,32)$ & & $(0,09 ; 0,61)$ & & $(0,09 ; 0,63)$ & \\
\hline & $\mathrm{C}$ & 1,05 & & 1,08 & & 0,82 & & 0,79 & \\
\hline & & $(0,65 ; 1,70)$ & & $(0,62 ; 1,88)$ & & $(0,47 ; 1,42)$ & & $(0,46 ; 1,36)$ & \\
\hline & $D-E$ & 1,00 & & 1,00 & & 1,00 & & 1,00 & \\
\hline
\end{tabular}


Tabela 3. continuação

\begin{tabular}{|c|c|c|c|c|c|c|c|c|c|}
\hline \multirow[b]{2}{*}{ Nível } & \multirow[b]{2}{*}{ Variáveis } & \multicolumn{4}{|c|}{ Masculino } & \multicolumn{4}{|c|}{ Feminino } \\
\hline & & $\begin{array}{c}\text { RP } \\
\text { bruta(IC }\left(\mathrm{IC}_{95 \%}\right)\end{array}$ & $\begin{array}{c}\text { Valor } \\
\text { p }\end{array}$ & $\begin{array}{c}\text { RP ajustada } \\
\left(\text { IC }_{95 \%}\right)\end{array}$ & $\begin{array}{c}\text { Valor } \\
\text { p }\end{array}$ & $\begin{array}{c}\text { RP } \\
\text { bruta }\left(\mathrm{IC}_{95 \%}\right)\end{array}$ & $\begin{array}{c}\text { Valor } \\
\text { p }\end{array}$ & $\begin{array}{c}\text { RP ajustada } \\
\left(\mathrm{IC}_{95 \%}\right)\end{array}$ & $\begin{array}{c}\text { Valor } \\
\mathrm{p}\end{array}$ \\
\hline \multirow[t]{5}{*}{1} & $\begin{array}{l}\text { Escolaridade da } \\
\text { mãe (anos } \\
\text { completos) }\end{array}$ & & $0,999^{\mathrm{b}}$ & & $0,491^{\mathrm{b}}$ & & $0,270^{\mathrm{b}}$ & & $0,980^{\mathrm{b}}$ \\
\hline & 0 a 4 & 1,00 & & 1,00 & & 1,00 & & 1,00 & \\
\hline & 5 a 8 & $\begin{array}{c}0,99 \\
(0,52 ; 1,88)\end{array}$ & & $\begin{array}{c}1,21 \\
(0,55 ; 2,67)\end{array}$ & & $\begin{array}{c}1,52 \\
(0,82 ; 2,79)\end{array}$ & & $\begin{array}{c}1,00 \\
(0,45 ; 2,23)\end{array}$ & \\
\hline & 9 a 11 & $\begin{array}{c}0,99 \\
(0,51 ; 1,91)\end{array}$ & & $\begin{array}{c}1,33 \\
(0,61 ; 2,89)\end{array}$ & & $\begin{array}{c}1,30 \\
(0,66 ; 2,58)\end{array}$ & & $\begin{array}{c}0,94 \\
(0,41 ; 2,16)\end{array}$ & \\
\hline & 12 ou mais & $\begin{array}{c}0,94 \\
(0,35 ; 2,49)\end{array}$ & & $\begin{array}{c}0,53 \\
(0,15 ; 1,91)\end{array}$ & & $\begin{array}{c}2,20 \\
(0,97 ; 5,00)\end{array}$ & & $\begin{array}{c}0,80 \\
(0,25 ; 2,54)\end{array}$ & \\
\hline \multirow[t]{5}{*}{1} & $\begin{array}{l}\text { Idade da mãe } \\
\text { (anos) }\end{array}$ & & $0,896^{\mathrm{b}}$ & & $0,896^{\mathrm{b}}$ & & $0,310^{\mathrm{b}}$ & & $0,723^{\mathrm{b}}$ \\
\hline & $<20$ & 1,00 & & 1,00 & & 1,00 & & 1,00 & \\
\hline & 20 a 29 & $\begin{array}{c}1,05 \\
(0,38 ; 2,90)\end{array}$ & & $\begin{array}{c}0,95 \\
(0,30 ; 3,00)\end{array}$ & & $\begin{array}{c}1,26 \\
(0,59 ; 2,70)\end{array}$ & & $\begin{array}{c}1,27 \\
(0,43 ; 3,75)\end{array}$ & \\
\hline & 30 a 34 & $\begin{array}{c}0,99 \\
(0,34 ; 2,90)\end{array}$ & & $\begin{array}{c}0,89 \\
(0,26 ; 3,05)\end{array}$ & & $\begin{array}{c}2,08 \\
(0,88 ; 4,96)\end{array}$ & & $\begin{array}{c}1,48 \\
(0,43 ; 5,10)\end{array}$ & \\
\hline & 35 ou mais & $\begin{array}{c}1,29 \\
(0,42 ; 3,91)\end{array}$ & & $\begin{array}{c}1,19 \\
(0,35 ; 4,07)\end{array}$ & & $\begin{array}{c}1,36 \\
(0,58 ; 3,20)\end{array}$ & & $\begin{array}{c}0,87 \\
(0,25 ; 3,00)\end{array}$ & \\
\hline \multirow[t]{3}{*}{1} & $\begin{array}{l}\text { Situação } \\
\text { conjugal }\end{array}$ & & $0,879^{\mathrm{b}}$ & & $0,964^{\mathrm{b}}$ & & $0,622^{\mathrm{b}}$ & & $0,003^{\mathrm{b}}$ \\
\hline & $\begin{array}{l}\text { Sem } \\
\text { companheiro }\end{array}$ & 1,00 & & 1,00 & & 1,00 & & 1,00 & \\
\hline & $\begin{array}{l}\text { Com } \\
\text { companheiro }\end{array}$ & $\begin{array}{c}1,01 \\
(0,90 ; 1,13)\end{array}$ & & $\begin{array}{c}1,00 \\
(0,90 ; 1,10)\end{array}$ & & $\begin{array}{c}1,02 \\
(0,94 ; 1,11)\end{array}$ & & $\begin{array}{c}1,37 \\
(1,11 ; 1,68)\end{array}$ & \\
\hline \multirow[t]{3}{*}{1} & $\begin{array}{l}\text { Cor da pele da } \\
\text { mãe }\end{array}$ & & $0,039^{\mathrm{b}}$ & & $0,063^{\mathrm{b}}$ & & $0,023^{\mathrm{b}}$ & & $0,076^{\mathrm{b}}$ \\
\hline & Branca & 1,00 & & 1,00 & & 1,00 & & 1,00 & \\
\hline & Não branca & $\begin{array}{c}0,60 \\
(0,37 ; 0,98)\end{array}$ & & $\begin{array}{c}0,60 \\
(0,33 ; 1,10)\end{array}$ & & $\begin{array}{c}0,58 \\
(0,36 ; 0,93)\end{array}$ & & $\begin{array}{c}0,61 \\
(0,35 ; 1,05)\end{array}$ & \\
\hline \multirow[t]{3}{*}{2} & $\begin{array}{l}\text { Trabalho fora } \\
\text { do lar }\end{array}$ & & $0,024^{\mathrm{b}}$ & & $0,094^{\mathrm{b}}$ & & $0,055^{\mathrm{b}}$ & & $0,806^{\mathrm{b}}$ \\
\hline & Sim & 1,00 & & 1,00 & & 1,00 & & 1,00 & \\
\hline & Não & $\begin{array}{c}0,58 \\
(0,36 ; 0,93)\end{array}$ & & $\begin{array}{c}0,60 \\
(0,33 ; 1,09)\end{array}$ & & $\begin{array}{c}0,64 \\
(0,40 ; 1,01)\end{array}$ & & $\begin{array}{c}0,93 \\
(0,52 ; 1,66)\end{array}$ & \\
\hline \multirow[t]{5}{*}{3} & $\begin{array}{l}\text { Índice de massa } \\
\text { corporal da mãe }\end{array}$ & & $0,002^{\mathrm{b}}$ & & $0,006^{\mathrm{b}}$ & & $0,329^{\mathrm{b}}$ & & $0,006^{\mathrm{b}}$ \\
\hline & Desnutrição & $\begin{array}{c}0,02 \\
(0,00 ; 0,16)\end{array}$ & & $\begin{array}{c}0,03 \\
(0,00 ; 0,23)\end{array}$ & & $\begin{array}{c}0,29 \\
(0,07 ; 1,14)\end{array}$ & & $\begin{array}{c}0,03 \\
(0,00 ; 0,23)\end{array}$ & \\
\hline & Eutrofia & 1,00 & & 1,00 & & 1,00 & & 1,00 & \\
\hline & Sobrepeso & $\begin{array}{c}0,99 \\
(0,59 ; 1,67)\end{array}$ & & $\begin{array}{c}0,82 \\
(0,44 ; 1,52)\end{array}$ & & $\begin{array}{c}1,04 \\
(0,61 ; 1,79)\end{array}$ & & $\begin{array}{c}0,81 \\
(0,43 ; 1,53)\end{array}$ & \\
\hline & Obesidade & $\begin{array}{c}1,27 \\
(0,72 ; 2,26)\end{array}$ & & $\begin{array}{c}1,07 \\
(0,49 ; 2,32)\end{array}$ & & $\begin{array}{c}1,10 \\
(0,58 ; 2,08)\end{array}$ & & $\begin{array}{c}1,27 \\
(0,63 ; 2,55)\end{array}$ & \\
\hline \multirow[t]{4}{*}{3} & $\begin{array}{l}\text { Circunferência } \\
\text { da cintura da } \\
\text { mãe }(\mathrm{cm})\end{array}$ & & $0,484^{\mathrm{b}}$ & & $0,764^{\mathrm{b}}$ & & $0,840^{\mathrm{b}}$ & & $0,501^{\mathrm{b}}$ \\
\hline & $<80$ & 1,00 & & 1,00 & & 1,00 & & 1,00 & \\
\hline & 80 a 87 & $\begin{array}{c}0,84 \\
(0,45 ; 1,59)\end{array}$ & & $\begin{array}{c}0,75 \\
(0,34 ; 1,64)\end{array}$ & & $\begin{array}{c}1,17 \\
(0,65 ; 2,10)\end{array}$ & & $\begin{array}{c}1,32 \\
(0,64 ; 2,71)\end{array}$ & \\
\hline & 88 ou mais & $\begin{array}{c}1,20 \\
(0,70 ; 2,06)\end{array}$ & & $\begin{array}{c}0,87 \\
(0,37 ; 2,00)\end{array}$ & & $\begin{array}{c}1,01 \\
(0,56 ; 1,85)\end{array}$ & & $\begin{array}{c}0,76 \\
(0,23 ; 2,45)\end{array}$ & \\
\hline
\end{tabular}

RP: Razão de prevalência; $\mathrm{IC}_{95 \%}$ : Intervalo de confiança de $95 \%$; ${ }^{a}$ Teste de Wald para tendência linear; ${ }^{\mathrm{b}}$ Teste de Wald para heterogeneidade; ${ }^{\mathrm{C}}$ As variáveis foram ajustadas para as do mesmo nível e para as do nível superior 
lar da mãe e excesso de peso das crianças. Por outro lado, estudo realizado por Jesus et al. ${ }^{34} \mathrm{evi}$ denciou similar associação em crianças com quatro meses de idade, embora não tenha sido controlado para possíveis fatores de confusão. Balaban e Silva ${ }^{35}$ sugerem que crianças cujas mães trabalham fora do lar são mais precocemente desmamadas e expostas ao consumo dos alimentos da família, estando mais susceptíveis ao sobrepeso. Além disso, essas mães teriam uma tendência a agradar os filhos com merendas, que na maioria das vezes apresentam alto valor calórico e baixo valor nutricional ${ }^{34}$. Ainda, segundo Fisher et al. ${ }^{36}$, os cuidadores de crianças tendem a subestimar mais o sobrepeso dos meninos do que o das meninas, fornecendo uma possível explicação da associação do trabalho fora do lar com o excesso de peso apenas nos meninos.

Harbaugh et al. ${ }^{37}$ também não evidenciaram associação entre cor da pele materna e excesso de peso em crianças, enquanto estudo realizado nos Estados Unidos encontrou maior prevalência de excesso de peso nos filhos de mulheres negras ${ }^{7}$.

No presente estudo, o nível socioeconômico permaneceu inversamente associado ao excesso de peso nas meninas, após ajuste para possíveis fatores de confusão. Alguns estudos mostraram resultados discordantes. Embora Zöllner e Fisberg $^{27}$ não tenham observado tal associação, outros autores ${ }^{7,38}$ evidenciaram associação positiva entre estas duas variáveis, porém nesses estudos, a análise não foi estratificada por sexo. Estudo de Monteiro ${ }^{39}$ sugere que o desenvolvimento econômico e a magnitude da obesidade não são fenômenos necessariamente relacionados, havendo países com níveis de riqueza semelhantes e prevalências muito distintas de obesidade.

Resultados encontrados em países desenvolvido ${ }^{25,40}$ mostraram que o tempo de amamentação exclusiva não foi associado ao excesso de peso em meninos e meninas de até três anos de idade, após análise ajustada. Outros autores ${ }^{41,42}$ também não evidenciaram tal associação em crianças com faixa etária de três a cinco anos, embora o tempo de amamentação avaliado incluiu o consumo de alimentos complementares. Entretanto, estudos de revisão realizados por Dewey ${ }^{43}$, Arenz et al. ${ }^{44} \mathrm{e}$ Owen et al. ${ }^{45}$ concluíram que o aleitamento materno protege da obesidade infantil. Todavia, tais resultados, aparentemente paradoxais, podem ser devidos a questões metodológicas ou, ainda, a diferenças nos padrões de amamentação e nos tipos de alimentos complementares introduzidos a partir das diferenças socioeconômicas entre as famílias ou entre os países. Além disso, destaca-se a importância de se estudar outras variáveis alimentares, como aquelas referentes à alimentação complementar.

Foi observado que o excesso de peso nas crianças está disseminado em todas as macrorregiões brasileiras, tanto na zona urbana quanto na rural, não estando associado a regiões mais ou menos desenvolvidas. Similarmente, a última Pesquisa de Orçamentos Familiares ${ }^{46}$ evidenciou alta prevalência de obesidade em todas as regiões brasileiras. Manios et al. ${ }^{47}$ não observaram diferença nas prevalências de excesso de peso entre crianças residentes nas zonas urbanas e rurais, embora não tenha sido realizada análise ajustada. Todavia, alguns autores evidenciaram que as crianças residentes em zonas urbanas apresentaram maiores prevalências de excesso de peso ${ }^{8,48}$. Esse padrão é, particularmente, atribuído às diferenças no acesso à alimentação e na atividade física nas áreas urbana e rural ${ }^{49}$.

Similarmente aos resultados encontrados no presente estudo, Plachta-Danielzki et al..$^{50}$ evidenciaram, em seu estudo realizado com crianças e adolescentes na Alemanha, que não houve relação entre escolaridade materna e excesso de peso, em ambos os sexos. Estudos realizados na Holanda e na Grécia com crianças de até cinco anos de idade também não evidenciaram essa associação ${ }^{47,51}$. Manios et al. ${ }^{47}$ sugerem que a escolaridade materna não está associada ao excesso de peso em crianças mais jovens, somente em crianças com mais idade. Tais estudos mostram resultados similares, embora tenham sido realizados em diferentes regiões do mundo.

Não foi evidenciada associação entre excesso de peso nas crianças e as variáveis idades da criança e da mãe. Vitolo et al. ${ }^{52}$, em seu estudo realizado com crianças de até cinco anos de idade em São Leopoldo (RS), não observaram associação entre o excesso de peso e a idade das crianças. Diferentemente, Gewa ${ }^{8}$ e Vieira et al $^{53}$ observaram menores prevalências de excesso de peso à medida que aumenta a idade da criança. Quanto à idade materna, Kitsantas e Gaffney ${ }^{7}$, Vitolo et al. ${ }^{52}$ e Dubois e Girard ${ }^{54}$ não encontraram associação entre essa variável e o excesso de peso em crianças de até cinco anos de idade.

Um ponto a ser considerado no presente estudo é referente às diferentes metodologias utilizadas para definir, categorizar e avaliar o estado nutricional de crianças. Tal fato dificulta a comparação com outros estudos que avaliaram os fatores associados ao excesso de peso infantil.

Conclui-se que o principal achado do presente estudo foi a forte e significativa associação 
positiva entre o peso ao nascer e o excesso de peso das crianças. Outros achados relevantes foram as associações entre a obesidade da mãe e o excesso de peso das crianças de ambos os sexos. Além disso, os meninos amamentados por um período de 5 a 6 meses apresentaram menor prevalência de excesso de peso; enquanto que, nas meninas observou-se uma associação inversa com o nível socioeconômico. Ademais, aquelas cujas mães viviam com companheiro foram as que apresentaram maior prevalência de excesso de peso.

Tendo em vista que, já na infância, a prevalência de sobrepeso vem aumentando de forma intensa, a melhor maneira de intervir é prevenir o mais precocemente possível, uma vez que o sobrepeso nessa fase da vida pode se projetar na adolescência e se perpetuar na vida adulta, tendo como consequências uma série de comorbidades $^{14,15}$. Salienta-se também, a necessidade de mais estudos utilizando delineamentos, preferencialmente longitudinais, que permitam avaliar os determinantes do sobrepeso infantil. Além disso, enfatiza-se a importância de programas de intervenção focados na família, uma vez que variáveis maternas, tanto biológicas quanto comportamentais, possuem um importante papel no desenvolvimento do excesso de peso na criança.

\section{Colaboradores}

CLP Araújo e SW Madruga contribuíram na revisão e correção do artigo. FO Meller participou de todas as etapas da produção do artigo. 


\section{Referências}

1. World Health Organization (WHO). Obesity: preventing and managing the global epidemic. Geneva: WHO; 1997. (Report of a WHO Consultation on Obesity).

2. Coutinho JG, Gentil PC, Toral N. Malnutrition and obesity in Brazil: dealing with the problem through a unified nutritional agenda. Cad Saude Publica 2008; 24(Supl. 2):332-340.

3. Batista Filho M, Rissin A. A transição nutricional no Brasil: tendências regionais e temporais. Cad Saude Publica 2003; 19(Supl. 1):181-191.

4. Deitel M. The International Obesity Task Force and "globesity". Obes Surg 2002; 12(5):613-614.

5. Pinheiro ARO, Freitas SFT, Corso ACT. Uma abordagem epidemiológica da obesidade. Rev Nutr 2004; 17(4):523-533.

6. Loaiza S, Coustasse A, Urrutia-Rojas X, Atalah E. Birth weight and obesity risk at first grade in a cohort of Chilean children. Nutr Hosp 2011; 26(1):214-219.

7. Kitsantas P, Gaffney K. Risk profiles for overweight/ obesity among preschoolers. Early Hum Dev 2010; 86(9):563-568.

8. Gewa CA. Childhood overweight and obesity among Kenyan pre-school children: association with maternal and early child nutritional factors. Public Health Nutr 2009; 13(4):496-503.

9. Editorial. Childhood obesity: an emerging publichealth problem. The Lancet 2001; 357(9273):19892066.

10. de Onis M, Blössner M, Borghi E. Global prevalence and trends of overweight and obesity among preschool children. Am J Clin Nutr 2010; 92(5):12571264.

11. Garipagaoglu M, Budak N, Süt N, Akdikmen O, Oner N, Bundak R. Obesity risk factors in Turkish children. J Pediatr Nurs 2009; 24(4):332-337.

12. Abrantes MM, Lamounier JA, Colosimo EA. Prevalência de sobrepeso e obesidade em crianças e adolescentes das regiões Sudeste e Nordeste. J Pediatr 2002; 78(4):335-340.

13. Brasil. Ministério da Saúde (MS). Cadernos de Atenção Básica, no 12. Brasília: MS; 2006. (Série A - Obesidade)

14. World Health Organization (WHO). Obesity: preventing and managing the global epidemic. WHO Technical Report Series 894. Geneva: WHO; 2000.

15. Muller MJ, Mast M, Asbeck I, Langnase K, Grund A. Prevention of obesity - is it possible? Obes Rev 2001; 2(1):15-28.

16. Dietz WH, Gortmaker SL. Preventing obesity in children and adolescents. Annu Rev Public Health 2001; 22:337-353.
17. Guedes DP, Paula IG, Guedes JERP, Stanganelli LCR. Prevalência de sobrepeso e obesidade em crianças e adolescentes: estimativas relacionadas ao sexo, à idade e à classe socioeconômica. Rev Bras Educ Fís Esp 2006; 20(3):151-163.

18. Brasil. Ministério da Saúde (MS). Relatório Final da Pesquisa Nacional de Demografia e Saúde. Brasília: Pesquisa Nacional de Demografia e Saúde da Criança e da Mulher; [página na Internet] 2006. [acessado 2013 dez 27]. Disponível em: http://www. saude.gov.br/pnds2006

19. World Health Organization (WHO). Physical Status: the Use and Interpretation of Anthropometry. Geneva: WHO; 1995.(Report of a WHO Expert Committee. WHO Technical Report Series, n. ${ }^{\circ} 854$ )

20. Census and Survey Processing System (CENSUS). US Census Bureau. Washington; [página na Internet] 2000. [acessado $2013 \mathrm{dez} 27]$. Disponível em: http://www.census.gov

21. World Health Organization (WHO). WHO Child Growth Standards: length/height-for-age, weight-forage, weight-for-length, weight-for-height and body mass index-for-age: methods and development. Geneva: WHO; 2006.

22. Associação Brasileira de Empresas de Pesquisa (ABEP). Critério de classificação econômica Brasil 2011. [página na Internet]. [acessado $2013 \mathrm{dez} 27]$. Disponível em: http://www.abep.org/novo/Content. aspx?ContentID $=302$

23. World Health Organization (WHO). WHO Growth reference data for 5-19 years. Geneva: WHO; 2007.

24. Victora CG, Huttly SR, Fuchs SC, Olinto MT. The role of conceptual frameworks in epidemiological analysis: a hierarchical approach. Int J Epidemiol 1997; 26(1):224-227.

25. Weyermann M, Rothenbacher D, Brenner H. Duration of breastfeeding and risk of overweight in childhood: a prospective birth cohort study from Germany. Int J Pediatr Obes 2006; 30(8):1281-1287.

26. Oldroyd J, Renzaho A, Skouteris H. Low and high birth weight as risk factors for obesity among 4 to 5 year-old Australian children: does gender matter? Eur J Pediatr 2011; 170(7):899-906.

27. Zöllner CC, Fisberg RM. Estado nutricional e sua relação com fatores biológicos, sociais e demográficos de crianças assistidas em creches da Prefeitura do Município de São Paulo. Rev Bras Saude Matern Infant 2006; 6(3):319-328.

28. Chaparro MP, Langellier BA, Kim LP, Whaley SE. Predictors of accurate maternal perception of their preschool child's weight status among Hispanic WIC participants. Obesity (Silver Spring) 2011; 19(10): 2026-2030. 
29. Jingxiong J, Rosenqvist U, Huishan W, Koletzko B, Guangli L, Jing H, Greiner T. Relationship of parental characteristics and feeding practices to overweight in infants and young children in Beijing, China. Public Health Nutr 2008; 12(7):973-978.

30. Ong KK. Size at birth, postnatal growth and risk of obesity. Horm Res 2006; 65(Supl. 3):65-69.

31. Perez-Pastor EM, Metcalf BS, Hosking J, Jeffery AN, Voss LD, Wilkin TJ. Assortative weight gain in mother-daughter and father-son pairs: an emerging source of childhood obesity. Longitudinal study of trios (EarlyBird 43). Int J Obes (Lond) 2009; 33(7):727-735.

32. Zwiauer KF. Prevention and treatment of overweight and obesity in children and adolescents. Eur J Pediatr 2000; 159(Supl. 1):56-68.

33. Morrissey TW, Dunifon RE, Kalil A. Maternal employment, work schedules, and children's body mass index. Child Dev 2011; 82(1):66-81.

34. Jesus GM, Vieira GO, Vieira TO, Martins CC, Mendes CMC. Fatores determinantes do sobrepeso em crianças menores de 4 anos de idade. J Pediatr 2010; 86(4):311-316.

35. Balaban G, Silva GA. Efeito protetor do aleitamento materno contra a obesidade infantil. J Pediatr 2004; 80(1):7-16.

36. Fisher L, Fraser J, Alexander C. Caregivers' inability to identify childhood adiposity: a cross-sectional survey of rural children and their caregivers' attitudes. Aust J Rural Health 2006; 14(2):56-61.

37. Harbaugh BL, Bounds W, Kolbo J, Molaison E, Zhang L. Prevalence estimates of overweight in head start preschoolers. J Pediatr Nurs 2009; 24(5):350-359.

38. Shrewsbury V, Wardle J. Socioeconomic status and adiposity in childhood: a systematic review of crosssectional studies 1990-2005. Obesity 2008; 16(2):275284.

39. Monteiro CA. Epidemiologia da obesidade. In: Halpern A, Matos AFG, Suplicy HL, Mancini MC, Zanella MT, editores. Obesidade. São Paulo: Lemos; 1998. p. 55-56.

40. Durmu ${ }^{\circ}$ B, Van Rossem L, Duijts L, Arends LR, Raat H, Moll HA, Hofman A, Steegers EA, Jaddoe VW. Breast-feeding and growth in children until the age of 3 years: the Generation R Study. Br J Nutr 2011; 105(11):1704-1711.

41. Hediger ML, Overpeck MD, Kuczmarski RJ, Ruan WJ. Association between infant breastfeeding and overweight in young children. JAMA 2001; 285(19): 2453-2460.

42. Huus K, Ludvigsson JF, Enskär K, Ludvigsson J. Exclusive breastfeeding of Swedish children and its possible influence on the development of obesity: a prospective cohort study. BMC Pediatr 2008; 8:42.

43. Dewey KG. Is breastfeeding protective against child obesity? J Hum Lact 2003; 19(1):9-18.
44. Arenz S, Ruckerl R, Koletzko B, Von Kries R. Breastfeeding and childhood obesity - a systematic review. Int J Obes Relat Metab Disord 2004; 28(10):12471256.

45. Owen CG, Martin RM, Whincup PH, Smith GD, Cook DG. Effect of infant feeding on the risk of obesity across the life course: a quantitative review of published evidence. Pediatrics 2005; 115(5):1367-1377.

46. Instituto Brasileiro de Geografia e Estatística (IBGE). Antropometria e Estado Nutricional de Crianças, Adolescentes e Adultos no Brasil. Pesquisa de Orçamentos Familiares 2008-2009. Rio de Janeiro: IBGE; 2010.

47. Manios Y, Costarelli V, Kolotourou M, Kondakis K, Tzavara C, Moschonis G. Prevalence of obesity in preschool Greek children, in relation to parental characteristics and region of residence. BMC Public Health 2007; 7:178.

48. Apfelbacher CJ, Loerbroks A, Cairns J, Behrendt H, Ring J, Krämer U. Predictors of overweight and obesity in five to seven-year-old children in Germany: results from cross-sectional studies. $B M C$ Public Health 2008; 8:171.

49. Wang Y. Cross-national comparison of childhood obesity: the epidemic and the relationship between obesity and socioeconomic status. Int J Epidemiol 2001; 30(5):1129-1136.

50. Plachta-Danielzik S, Landsberg B, Johannsen M, Lange D, Müller MJ. Determinants of the prevalence and incidence of overweight in children and adolescents. Public Health Nutr 2010; 13(11):1870-1881.

51. Twells L, Newhook LA. Can exclusive breastfeeding reduce the likelihood of childhood obesity in some regions of Canada? Can J Public Health 2010; 101(1): 36-39.

52. Vitolo MR, Gama CM, Bortolini GA, Campagnolo PDB, Drachler ML. Some risk factors associated with overweight, stunting and wasting among children under 5 years old. J Pediatr 2008; 84(3):251-257.

53. Vieira MFA, Araújo CLP, Hallal PC, Madruga SW, Neutzling MB, Matijasevich A, Leal CMA, Menezes AMB. Estado nutricional de escolares de $1^{a}$ a $4^{a}$ séries do Ensino Fundamental das escolas urbanas da cidade de Pelotas, Rio Grande do Sul, Brasil. Cad Saude Publica 2008; 24(7):1667-1674.

54. Dubois L, Girard M. Early determinants of overweight at 4.5 years in a population-based longitudinal study. Int J Obes 2006; 30(4):610-617.

Artigo apresentado em 18/01/2013

Aprovado em 15/03/2013

Versão final apresentada em 20/03/2013 